\title{
Editorials
}

\section{The fournal of Clinical Pathology goes online (http://www.jclinpath.com)!}

The presence of the Internet is rapidly changing the world of scientific publication. Time spent previously in visiting libraries and chasing references is a thing of the past. Large electronic databases have become available over the Internet and have dramatically increased the accessibility of world literature. Because much of the scientific population has direct Internet access, relevant rapid and complete literature searches have fast become the norm. During the past few years, several of the larger journals have progressed electronically to become available "online". We are proud to announce that $\mathcal{F C P}$ is joining this important development and is now available online via HighWire, a division of the Stanford University's Green Library, which hosts many journals including those of the BMJ Publishing Group. Of course, $\mathcal{F C P}$ already had a webpage at the BMJ Publishing Group site but with limited options. The new website at the same address (www.jclinpath.com) has moved to California and is a different world.

The online journals have many added features of value. One of these benefits is that all publishers involved agree to share "toll free links". This means that references to any of the more than 200 journals hosted by HighWire link directly to the full text of that article without additional subscription charges. Furthermore, the BMJ Publishing Group aims to join the "CrossRef" initiative, whereby references will link directly to articles in participating journals regardless of where they are hosted. Access to the full text is determined in such cases by the individual publisher.

References to non-HighWire journals link directly to Medline where available, giving direct access to abstracts, and we are working towards similar links to ISI's Current Contents. These hyperlinks are just one of the ways to exploit the rapidly growing mass of information available online. $\mathcal{F C P}$ online contains full text articles plus tables and figures. These are published at the same time as the paper version, and so may be available some time before the paper journal arrives by post. Subscribers to the paper version of $\mathcal{F C P}$ and members of the Association of Clinical Pathologists will receive $\mathcal{F C P}$ online without additional costs. Institutional subscribers may subscribe to $\mathcal{F C P}$ online separately, the price depending on the size of the institution. Within $\mathcal{F C P}$ on-
About

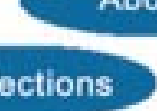

ns

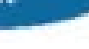

add "web-extra" material such as (more) colour figures, more extensive tables, animations, three dimensional pictures and films. Email alerts will automatically alert one to the contents list as a new issue becomes available, and these will soon be customisable to a particular area of interest. The "Collections" feature allows one to search quickly areas and this feature links directly with the BMJ and other specialist journals in the BMJ Publishing Group.

There will be quick links to related articles and articles citing $\mathcal{F C P}$ papers, as well as many useful links to related or interesting webpages. Accepted papers will be listed, and submitting an Web extra for material in subspecialty

"eLetter" allows quick response to and discussion about published manuscripts. There will be more options to enhance the open review process that we favour. ${ }^{1}$

$\mathcal{F C P}$ online will facilitate several other developments. A new manuscript tracking system will soon be implemented. This will allow electronic submission and reviewing of manuscripts, which will help to reduce further our manuscript turnaround time. It may well be possible for limited accessibility to the system to be provided for those submitting papers, so that authors can follow the flow of their manuscript through the editorial process. Furthermore, a cross journal reviewer database will soon be implemented. This will simplify finding suitable available reviewers, will not only increase journal efficiency, but will benefit expert reviewers who will no longer need to suffer the receipt of papers from different journals simultaneously. Finally, Pathology Interactive, our educational interactive CD$\mathrm{ROM},{ }^{2}$ will later also be integrated within the webpage. This will increase its availability, simplify its use, and present new interactive learning possibilities with better and faster integration with online literature databases. It will also obviate the current need to distribute CD-ROMs by mail. This improved access supports our view that specialist journals such as $\mathcal{F C P}$ can play an important role in continuing professional development. ${ }^{34}$

For the foreseeable future, there will be no change to access to the paper version of $\mathcal{F C P}$. It will still be made available to all subscribers. This is obviously important to those without direct online access and laser printers, or to 
those who simply prefer to read from a printed journal rather than from a screen.

We think this important new development puts $\mathcal{F C P}$ in company with many good journals that are now available electronically and places it on solid ground within the rapidly changing world of electronic scientific publishing.

Please visit our website at www.jclinpath.com and bookmark it!
PAUL J VAN DIEST HELEN HOLZEL

pj.vandiest@azvu.nl

1 Holzel H, Diest PJ van. The new millennium: time for a change! $f$ Clin Pathol 2000;53:1-2.

2 Holzel H, Diest PJ van, Heard S. Continuing professional development: is the future Pathology Interactive? F Clin Pathol 1999;52:401-2.

3 Bosman FT. Continuous professional development in pathology: a continental view. F Clin Pathol 2000;53:10-12.

4 Du Boulay C. Continuing professional development: some new perspectives. F Clin Pathol 1999;52:162-4. 